\section{OP76 HOUSEHOLDS AND HEALTH: HOW SMALL CLUSTERS CAN INFLUENCE RESULTS}

doi:10.1136/jech-2012-201753.076

AH Leyland, L Gray. Social and Public Health Sciences Unit, Medical Research Councill Chief Scientist Office, Glasgow, Scotland

Background There have been studies suggesting that various measures of health, health behaviours or health seeking cluster within households. Previous research on the impact of omitting small clusters from analyses have suggested little impact on parameter estimates or standard errors but have typically assumed small intraclass correlations - not usually the case for the household. Since there are typically few individuals per household, and many households contain just one person, several studies have excluded household from the analysis. We examine what difference it makes in practice when the level of household is excluded for a variety of outcomes.

Methods 7901 adults from 5063 households in 356 small areas were interviewed in the 2003 Scottish Health Survey; in 2512 $(49.6 \%)$ of households just one adult participated. We analysed systolic blood pressure (SBP), BMI, current smoking status, eating $5+$ portions of fruit and vegetables per day, eating oily fish at least once per week, and having seen a GP within the past 2 weeks. All results were adjusted for age and sex; in addition, we examined the effects of education, social class and area deprivation. Interactions were fitted between sex and all terms in the fixed and random parts of the model. Multiple imputation was used to account for item nonresponse. Multilevel linear and logistic regression models including and excluding household were fitted by Markov chain Monte Carlo and the results compared, with population average estimates obtained for logistic regression models.

Results The extent of clustering varied by outcome and gender; the proportion of the total unexplained variance at the household level ranged from $8.6 \%$ (95\% Credible interval 0.3-24.5) for systolic blood pressure for women to $87.5 \%$ (83.7-90.7) for eating oily fish for women. Bias was evident in both the fixed parameter estimates and their standard errors. The bias in the fixed parameters tended to be higher for outcomes for which the household variance was higher.

Conclusion For all outcomes the inclusion of household provided an improvement in model fit. Excluding the level of household led to biases not just in the standard errors but also in the parameter estimates themselves. This has implications not only regarding the need to account for clustering within households but also regarding the use of "sandwich estimators" that adjust the standard error but not the regression coefficient.

\section{Public Health Policy Analysis}

\section{OP77 TYPE A BEHAVIOUR PATTERN AND CORONARY HEART DISEASE: PHILIP MORRIS' “CROWN JEWEL”}

doi:10.1136/jech-2012-201753.077

M Petticrew, K Lee, M McKee. Faculty of Public Health and Policy, LSHTM, London, UK

Background The Type A Behaviour Pattern (TABP) - characterised as individuals who are highly competitive, time-conscious and aggressive - has been the subject of research for over fifty years. The concept was developed in the 1950s by U.S. cardiologists Meyer Friedman and Ray Rosenman who argued that TABP was a risk factor for coronary heart disease (CHD), notably among white middleclass males. The theory was initially supported by findings from the Western Collaborative Group study (WCGS) and the Framingham study. However, subsequent studies found no consistent evidence that TABP predicts CHD onset or outcome. This pattern of early positive findings followed by negative findings (a "decline effect") has long been a puzzle for TABP researchers. Despite this, the concept of Type A behaviour has continued to enjoy public appeal, fostered through popular books by Friedman and Rosenman which describe "how to recognise the deadly Type A pattern in your own personality". TABP has also remained the subject of contemporary public health research, and it features in discussions on the psychosocial causes of health inequalities. We analysed tobacco industry documents to show that Philip Morris and RJ Reynolds were major funders of TABP research, with selected positive findings used to counter concerns regarding tobacco and health.

Methods The Legacy Tobacco Documents Library (http://legacy. library.ucsf.edu) was systematically searched from 1959 to 2011 to identify relevant documents. The names of key individuals were identified through retrieved documents using a snowballing technique.

Results Our document analysis suggests that, in the case of TABP, the decline effects described above may be explained by the influence of tobacco industry funding. Phillip Morris channeled significant funds for TABP research through Duke and Yale Universities, including part-funding the Framingham study. It also funded a 10-year clinical trial of the effectiveness of counselling to reduce TABP, which it referred to as its "Crown Jewel", and funded Meyer Friedman's Institute to a total of almost US\$11million. A history of the relationship between TABP research and the Tobacco Industry will be presented. Conclusion It has not previously been shown that research into TABP was strongly influenced by the Tobacco Industry. This analysis extends further our understanding of the extent to which the tobacco industry has shaped major themes in contemporary public health research. Our findings also help explain the inconsistencies in the findings of epidemiological studies into TABP and mortality.

\section{OP78 SCIENTISTS, COMPETING INTERESTS, AND THE MEDIA: A CONTENT ANALYSIS OF UK NEWSPAPER REPORTING IN H1N1 INFLUENZA}

doi:10.1136/jech-2012-201753.078

${ }^{1} \mathrm{KNK}$ Chan, ${ }^{2} \mathrm{~S}$ O'Neill, ${ }^{3} \mathrm{KL}$ Mandeville. 'Department of Medicine, Barts and the London School of Medicine and Dentistry, London, UK; ${ }^{2}$ Department of Medicine, Imperial College London, London, UK; ${ }^{3}$ Global Health and Development, LSHTM, London, UK

Background Concerns were raised about the competing interests amongst scientists on advisory committees during the 2009/10 $\mathrm{H} 1 \mathrm{~N} 1$ influenza pandemic, particularly given the substantial public expenditure on antiviral drugs and vaccines. The media is known to strongly influence public demand for new drugs and policy decisions, and many scientists commented on the use of pharmaceutical products/emerging health risks during the $2009 \mathrm{H} 1 \mathrm{~N} 1$ pandemic in the UK media. This study assessed competing interests for the scientists promoting or rejecting the use of antivirals and/or vaccines in newspaper articles during the early pandemic.

Methods We performed a retrospective content analysis of United Kingdom national newspaper articles on H1N1 influenza published during the period when the government decided its policy on antiviral/vaccine provision (20 April and 5 July 2009). Two reviewers coded 436 articles independently and identified those scientists promoting or rejecting the use of antivirals/vaccine according to a prespecified protocol. Competing interests for these named scientists were then identified through a systematic search for potential or previously declared interests.

Results One in two scientists commenting on the use of antiviral/ vaccines in H1N1 influenza had undisclosed competing interests. Potential competing interests were identified in 6 of the 9 scientists $(66.7 \%)$ promoting vaccine use; 6 of the 10 scientists (60\%) promoting antivirals; and 1 of 4 scientists (25\%) rejecting antiviral use. The nature of these competing interests ranged from study funding to directorships of pharmaceutical companies. Only three articles made clear that the scientist concerned had a link with pharmaceutical companies. 
Conclusion This study showed substantial competing interests amongst scientists commenting on the use of antivirals and/or vaccines in H1N1 influenza during the period the UK government was deciding its pharmaceutical policy. Since commentaries in the media provide an alternative route for external pressure on health policy decisions, scientists should declare any potential competing interests for media interviews as for journal articles.

\section{OP79 YOUTH EXPOSURE TO TELEVISION ALCOHOL ADVERTISING IN THE UK}

doi:10.1136/jech-2012-201753.079

${ }^{1} \mathrm{~S}$ Patil, ${ }^{2} \mathrm{M}$ N Elliott, 'E M Winpenny, 'C Rohr, 'E Nolte. 'N/A, RAND Europe, Cambridge, UK; ${ }^{2} N / A$, RAND Corporation, Santa Moica, CA, USA

Background Increasing evidence suggests that exposure to alcohol marketing increases the likelihood that adolescents will start to drink alcohol and that among those who already consume alcohol exposure to alcohol advertising is likely to increase its use. Existing research in Europe has tended to focus on the content of alcohol marketing and advertising but our understanding of the actual levels of exposure remains inadequate. This study aims to contribute to filling this gap by analysing youth exposure to television alcohol advertising in the UK.

Methods We obtained data on viewership and on alcohol advertising volume for the top ten television channels with the highest number of viewers in the UK by age (4-9, 10-15, 16-24, 25 plus) for December 2010 to May 2011. Data were analysed descriptively to characterise youth viewership by channel, month and time of the day ('daypart') and alcohol advertising exposure. We then used a negative binomial regression model to measure incidental youth exposure to alcohol advertising relative to adults over 25 years of age. We applied sensitivity analyses to test robustness of the model. Results Viewership and alcohol advertising volume varied substantially across the 10 channels, months and dayparts. Children and adolescents (10-15 years) constituted about $4 \%$ of the total audience and young people (16-24 years) 8\%, yet their exposure to alcohol commercials was $5 \%$ and $50 \%$, respectively. Relative to adults 25 years of age and older, the regression analysis found advert intensity was higher where children and adolescents constituted a greater proportion of the viewership (incident rate ratio (IRR) 1.09, $95 \%$ CI 1.02, 1.17, $p=0.013$ ). This relationship was particularly strong for commercials of beer and cider (IRR $=1.14,95 \%$ CI 1.06 , $1.23, \mathrm{p}=0.000)$, super market brands (IRR $=1.16,95 \%$ CI 1.07, 1.28, $\mathrm{p}=0.000$ ), and ready-mix drinks (IRR $=1.51,95 \%$ CI 1.27, 1.78, $p=0.000$ ). In contrast, although the IRR for those aged 16 to 24 years also differed significantly from that observed for older adults, the difference was small $(1.02,95 \%$ CI 1.01, 1.04, $p=0.003)$. There were no statistically significant associations for wine and spirit advert incidence and viewership for any of the younger population groups.

Conclusion Our findings suggest that young people in the UK have a disproportionately higher exposure to television alcohol (except wine and spirits) advertising than would be expected from general viewership patterns. Alcohol advertising practices should be modified to limit exposure of underage viewers.

\section{OP80 MINIMUM UNIT PRICING FOR ALCOHOL: A DOCUMENT ANALYSIS OF EVIDENCE SUBMISSIONS TO THE SCOTTISH PARLIAMENT}

doi:10.1136/jech-2012-201753.080

'S V Katikireddi, ${ }^{2}$ S Hilton, 'L Bond. 'Evaluating the Health Effects of Social Interventions programme, MRC/CSO Social and Public Health Sciences Unit, Glasgow, UK; ${ }^{2}$ Understandings and Uses of Public Health Research, MRC/CSO Social and Public Health Sciences Unit, Glasgow, UK
Background Minimum unit pricing (MUP) of alcohol is a novel policy intervention aimed at increasing the price of the cheapest forms of alcohol to reduce alcohol consumption and associated harms. In Scotland, alcohol-related harms have increased exponentially during the past few decades and Scotland currently experiences the greatest level of alcohol-related mortality within the UK. Scotland would be the first country in the world to introduce the measure and therefore both advocates and critics have seized the opportunity to put forward the different arguments in the case of MUP. This study examines the evidence submitted to the Scottish Parliament with the aim of examining how arguments for and against MUP have been framed and to consider what forms of evidence have been drawn upon by different policy stakeholders to debate MUP.

Methods The Scottish Parliament received evidence submissions from a wide variety of stakeholders in its first consideration of MUP as part of the Stage 1 scrutiny process for the Alcohol (etc.) Bill 2010. Sixty-five documents submitted by 47 different stakeholders (including politicians, health professionals, industry representatives, supermarkets and researchers) considered within meetings of the Select Committee were analysed. Data management was assisted using Nvivo 9, framework matrices, and thematically analysed using constant comparative methods.

Results Arguments around alcohol policy have been actively constructed as a health issue by advocates for MUP in comparison to critics who highlight economic, ideological, and social aspects. Constructions of alcohol overconsumption as a population health issue, rather than an individual-level health problem, were contested. Alcohol epidemiology was drawn upon by advocates (to support a whole population approach) but also by critics (to argue alcohol harms are reducing). Issues raised in opposition include important potential secondary impacts such as exacerbating inequalities, increasing cross-border and illicit trade and perverse incentives (such as increases in profitability driving retailers to encourage increased sales).

Conclusion Framing of alcohol policy as a health (rather than, for example, trade or justice) issue that requires a whole population approach has been important in creating conditions amenable to MUP being seriously considered by policymakers. The initial framing of an issue in different ways can lead to similar sources of evidence, such as routine epidemiological data, being used to suggest different actions are appropriate. Public health researchers and practitioners should be aware of the active construction process occurring in policy definition and adapt research and advocacy accordingly.

\section{Public Health Interventions: Diet}

\section{OP81 ESTIMATING THE POTENTIAL OF POPULATION LEVEL CHANGES IN CHOLESTEROL AND BLOOD PRESSURE FOR REDUCING UK CORONARY HEART DISEASE MORTALITY RATES: A NOVEL MODELLING APPROACH}

doi:10.1136/jech-2012-201753.081

${ }^{1} \mathrm{M}$ O'Flaherty, ${ }^{2} \mathrm{P}$ Couch, ${ }^{2} \mathrm{M}$ Sperrin, ${ }^{2} \mathrm{~N}$ Green, ${ }^{2} \mathrm{~J}$ Ainsworth, ${ }^{3} \mathrm{M}$ Huffmann, ${ }^{3} \mathrm{D}$ LloydJones, ${ }^{2}$ Buchan, ${ }^{1} \mathrm{~S}$ Capewell. 'Deaprtment of Public Health and Policy, University of Liverpool, Liverpool, UK; ${ }^{2}$ Northwest Institute for Biohealth Informatics, University of Manchester,Manchester UK; ${ }^{3}$ Department of Preventive Medicine, Northwestern University, Chicago, USA

Background Coronary heart disease (CHD) mortality is declining. However, population ageing and increasing obesity and diabetes might reverse this trend. Strategies to reduce salt and saturated fat intake could substantially decrease population-wide cholesterol and blood pressure levels and consequently reduce CHD events. Our aim was to explore the potential reduction in CHD mortality 